OPEN ACCESS

Edited by:

Jai Prakash,

University of Twente, Netherlands

Reviewed by:

Sumit Arora,

University of South Alabama, USA

Yunbao Pan,

Jiangnan University, China

*Correspondence:

Monika Ehnman

monika.ehnman@ki.se

Specialty section:

This article was submitted to Cancer Molecular Targets and Therapeutics, a section of the journal

Frontiers in Oncology

Received: 03 August 2015

Accepted: 18 October 2015

Published: 04 November 2015

Citation:

Ehnman M and Larsson O (2015)

Microenvironmental targets in sarcoma.

Front. Oncol. 5:248.

doi: 10.3389/fonc.2015.00248

\section{Microenvironmental targets in sarcoma}

\author{
Monika Ehnman* and Olle Larsson \\ Department of Oncology-Pathology, Cancer Center Karolinska, Karolinska Institutet, Karolinska University Hospital, \\ Stockholm, Sweden
}

Sarcomas are rare malignant tumors affecting all age groups. They are typically classified according to their resemblance to corresponding normal tissue. Their heterogeneous features, for example, in terms of disease-driving genetic aberrations and body location, complicate both disease classification and development of novel treatment regimens. Many years of failure of improved patient outcome in clinical trials has led to the conclusion that novel targeted therapies are likely needed in combination with current multimodality regimens. Sarcomas have not, in contrast to the common carcinomas, been the subject of larger systematic studies on how tumor behavior relates to characteristics of the tumor microenvironment. There is consequently an urgent need for identifying suitable molecular targets, not only in tumor cells but also in the tumor microenvironment. This review discusses preclinical and clinical data about potential molecular targets in sarcomas. Studies on targeted therapies involving the tumor microenvironment are prioritized. A greater understanding of the biological context is expected to facilitate more successful design of future clinical trials in sarcoma.

Keywords: sarcoma, microenvironment, angiogenesis, stroma, targeted therapy, VEGF, PDGF, KIT

\section{INTRODUCTION}

Sarcomas represent rare malignant tumors of mesenchymal origin with more than 70 different entities (1). Multimodal treatment generally includes local control by surgery and/or radiation and systemic control by chemotherapy. Specific treatment protocols depend on clinical parameters, including stage classification, histological grade, tumor site, and subtype. Most of the sarcoma subtypes are relatively resistant to chemotherapy.

Diagnosis and treatment regimens should be carried out in a multidiscipline manner. It is therefore highly recommended that patient care is centralized to a sarcoma reference center immediately after the initial detection of a suspected sarcoma. The European Sarcoma Network Working Group has developed clinical practice guidelines for diagnosis, treatment, and follow-up in bone sarcomas (2),

\footnotetext{
Abbreviations: ASPS, alveolar soft part sarcoma; CSF1R, colony stimulating factor 1 receptor; DFSP, dermatofibrosarcoma protuberans; EGFR, epidermal growth factor receptor; EMA, European Medicines Agency; EWS/FLI, Ewing sarcoma breakpoint region 1; FDA, Food and Drug Administration; FGFR, fibroblast growth factor receptor; FLT3, fms-related tyrosine kinase 3; GIST, gastrointestinal tumor; KIT, v-kit Hardy-Zuckerman 4 feline sarcoma viral oncogene homolog; MET, hepatocyte growth factor receptor; PAX3-FOXO1, paired box 3-forkhead box O1; PDGFR, platelet-derived growth factor receptor; RAF, zinc fingers and homeoboxes 2; RANKL, tumor necrosis factor (ligand) superfamily, member 11; RECIST, response evaluation criteria in solid tumors; RET, ret proto-oncogene; STS, soft tissue sarcoma; TKI, tyrosine kinase inhibitor; VEGFR, vascular endothelial growth factor receptor; vWF, von Willebrand factor; WHO, World Health Organization.
} 
soft tissue, and visceral sarcomas (3), and gastrointestinal stromal tumors (GIST) (4).

Patient outcome is influenced by many parameters. Local tumor recurrence and metastatic spread at diagnosis are associated with a poor prognosis. Curative treatment options are limited in these patient groups. Better treatment options are also needed to reduce long-term complications for patients in remission. This is particularly important to consider in pediatric patients where established treatments of today are known to be associated with, e.g., reduced fertility later in life.

Targeted therapies are currently emerging as a promising complementary or alternative treatment in several pathological entities including GIST $(5,6)$. Attempts have also been made to identify patient subgroups that are likely to benefit from this novel type of treatment. Given the mechanistic nature of targeted therapies, a molecular target essential for disease progression of each individual tumor typically needs to be identified. Detailed knowledge on sarcoma biology is therefore important for clinical progress.

\section{SUBTYPE CLASSIFICATION IN SARCOMA}

Subtype classification in sarcoma is today guided by the 2013 World Health Organization (WHO) classification system (7). Dominating soft tissue sarcoma (STS) subtypes include undifferentiated pleomorphic sarcoma (UPS) and liposarcoma in adults and rhabdomyosarcoma in children. Also GIST is now a dominating subtype in adults after the inclusion in the soft tissue section. Common adult bone sarcomas include osteosarcoma and chondrosarcoma. Osteosarcoma is also a dominating subtype in children together with Ewing sarcoma. Examples of sarcoma subtypes according to their differentiation status are presented in Table 1.

Some of the entities are classified with the help of cytogenetics, or molecular genetics, searching for characteristic genetic aberrations including pathognomonic fusion genes. A large group of sarcomas is however currently being classified according to exclusion criteria when a more precise categorization fails. Many of these tumors are referred to as UPS, or earlier, malignant fibrous histiocytoma. It is reasonable to assume that the classification of tumors belonging to this latter group will further improve with an increased molecular understanding.

Inconsistent classification of sarcomas has complicated registry-based studies, both over time and between countries. With increased knowledge about sarcoma biology, subtype classification is likely to rely more on molecular hallmarks in combination with observations made by conventional histology and selected imaging assessments (9). This will allow more stringent analyses in defined patient groups and ultimately improve patient outcome.

\section{GENETIC ABERRATIONS OF COMMON SARCOMA SUBTYPES}

We are only in the beginning of understanding the genetic aberrations involved in sarcoma development and progression. It has
TABLE 1 | Schematic overview of sarcomas of soft and bone tissue.

\begin{tabular}{ll}
\hline Differentiation of soft tissue & Examples \\
sarcomas & \\
Adipocytic tumors & Dedifferentiated liposarcoma \\
Fibroblastic/myofibroblastic tumors & Fibrosarcoma \\
Smooth muscle tumors & Leiomyosarcoma \\
Pericytic (perivascular) tumors & Malignant glomus tumor \\
Skeletal muscle tumors & Embryonal rhabdomyosarcoma \\
Vascular tumors & Angiosarcoma \\
Chondro-osseous tumors & Extraskeletal chondrosarcoma \\
Gastrointestinal tumors & Gastrointestinal stromal tumor (GIST) \\
Nerve sheath tumors & Malignant peripheral nerve sheath tumor \\
Tumors of uncertain differentiation & Synovial sarcoma \\
Undifferentiated/unclassified & Undifferentiated pleomorphic sarcoma \\
sarcomas & (UPS) \\
Differentiation of bone sarcomas & Examples \\
Chondrogenic tumors & Chondrosarcoma grade II-III \\
Osteogenic tumors & Conventional high-grade osteosarcoma \\
Fibrogenic tumors & Fibrosarcoma \\
Osteoclastic giant cell rich tumors & Malignancy in giant cell tumor of bone \\
Notochordal tumors & Chordoma \\
Vascular tumors & Angiosarcoma \\
Myogenic tumors & Leiomyosarcoma \\
Lipogenic tumors & Liposarcoma \\
Miscellaneous tumors & Ewing sarcoma \\
&
\end{tabular}

Modified from the present WHO classification of tumors (8).

aDominating histotypes: UPS, liposarcoma (adults), rhabdomyosarcoma (children, young adults). Also GIST is now a dominating histotype in adults after the inclusion in the soft tissue section.

${ }^{b}$ Dominating histotypes: Osteosarcoma, chondrosarcoma (adults), osteosarcoma, Ewing sarcoma (children, young adults).

been recognized that genetic aberrations often lead to activation of tyrosine kinase growth factor receptors (10). However, distinct oncogenic drivers at the molecular level are often only detected in subsets of a defined sarcoma entity. The histology that traditionally aids sarcoma subtype classification is therefore, to our understanding today, only partially linked to the molecular profile of the tumor.

Specific genetic aberrations are commonly found in the class of sarcomas with a simple, or close to simple, karyotype. The confirmed presence of disease-driving, activating mutations in KIT or PDGFR $\alpha$ in GIST can today, e.g., be used to predict tumor recurrence and identify patients who are likely to benefit from adjuvant therapy (11).

Ewing sarcoma and alveolar rhabdomyosarcoma are both examples of translocation-associated sarcomas. The most common translocations involve transcription factors that actively dysregulate gene expression. EWS/FLI in Ewing sarcoma has been shown to upregulate the PDGFR ligand PDGF-C (12). The oncogenic fusion gene PAX3-FOXO1, found in the majority of all alveolar rhabdomyosarcomas, has been linked to poorer patient outcome $(13,14)$. Other examples of sarcomas with specific genetic aberrations involving chromosomal translocations are synovial sarcoma, clear cell sarcoma of soft tissue, myxoid, and round cell liposarcoma.

Many sarcomas are, however, known to have a more complex karyotype. Pleomorphic undifferentiated sarcoma, pleomorphic and dedifferentiated liposarcoma, leiomyosarcoma, pleomorphic rhabdomyosarcoma, osteosarcoma, and chondrosarcoma all 
belong to this other main class of sarcomas (10). The diseasedriving mechanisms of these tumors are likely to be related to defects in the cell cycle checkpoints and the genetic instability as such.

\section{THE SARCOMA TUMOR MICROENVIRONMENT}

The microenvironment of tumors is composed of multiple stromal cell types and extracellular matrix proteins in addition to the cancer cells. The stromal compartment is typically involved in structural and functional support of tumor growth and coevolves together with the tumor cells in a unique manner depending on tumor type and tumor stage. It had already been described in 1960 , how a primary tumor of sarcoma can evade the anticancer immune response by establishing an immune-privileged microenvironment $(15,16)$. In the 1970s, Juda Folkman proposed that angiogenesis is essential for solid tumor growth (17).

The importance of the tumor microenvironment for metastatic growth was addressed in the seed and soil theory (18). This theory describes the metastatic-prone tumor cell as "the seed" and the preferred metastatic site as "the soil." Another complementary theory is the anatomical-mechanical hypothesis (19) suggesting that tumor cells are rather passively shed from the primary tumor by lymphatic drainage and the vascular system. This would then result in metastatic growth at anatomically accessible sites defined by the location of the primary tumor.

Both theories on the metastatic process are today considered applicable in a tumor-specific context $(20,21)$. The importance of tumor cell intravasation into the vascular system has particularly been addressed in sarcoma, where vascular invasion, as defined by the presence of tumor cells within any space having an endothelial lining, has been identified as a prognostic factor for metastasis (22). In the study by Engellau et al., vascular invasion was detected in 50 of 140 STS and was shown to closely associate with necrosis and malignancy grade.

\section{Blood Vessel-Associated Cells, Angiogenesis, and Prognostic Biomarkers} Blood vessels in tumors are composed of endothelial cells and various amounts of supportive mural cells including pericytes and vascular smooth muscle cells. The most commonly used markers for endothelial cells are CD31, CD34, VEGFR2, factor VIII, vWF, and endoglin. Podocalyxin was used as a vessel marker in experimental rhabdomyosarcoma to confirm the antiangiogenic response to sorafenib (23). CD31 is often considered to be a panendothelial marker, whereas endoglin is expressed by activated endothelium. Endoglin is also expressed by tumor cells in Ewing sarcoma, where its expression has been shown to correlate with worse patient survival (24).

In 1999, Tomlinson et al. concluded that the pattern of angiogenesis is different between sarcomas and carcinomas (25). Their study showed that the capillaries in carcinomas are clustered in bursts within the tumor stroma and that the microvessel density in these bursts can be used as a prognostic factor. By contrast, microvessel density in sarcomas was shown to have a more homogeneous appearance. A more recent study confirmed this pattern of angiogenesis showing that hot spots of angiogenesis were diffuse in high-grade STS and only present in 33\% of the investigated specimens (26).

High microvessel density, as assessed by CD31 staining, has been shown to correlate with, e.g., high VEGF expression, tumor size $\geq 5 \mathrm{~cm}$ and high tumor grade in GIST (27). Microvessel density in other sarcomas has been evaluated, but occasionally also questioned in terms of inconsistently used methodologies and prognostic relevance $(26,28-32)$. Most studies on angiogenesis have chosen to focus on VEGF detection rather than microvessel density $(33,34)$. Both VEGF expression and circulating VEGF levels are of suggested clinical relevance in sarcomas. VEGF and additional markers of angiogenesis in sarcomas have been reviewed elsewhere $(33,35,36)$.

\section{Other Infiltrating Stromal Cell Types in Sarcoma}

The importance or prognostic relevance of infiltrating stromal cell types in sarcomas has not been extensively characterized. Some functions have been associated, directly or indirectly, with angiogenesis or vasculogenesis. Recruited CD34+ bone marrow stem cells have been shown to contribute to the growing tumor vasculature in response to VEGF in experimental Ewing sarcoma (37). M2-like, CD163+ CD14+ macrophages have been described in naïve ASPS. These tumor-associated macrophages of myeloid origin are believed to promote tumor progression and possibly VEGF-mediated vasculogenesis (38). In contrast, M1 macrophages are typically tumor preventing and respond to interferon- $\gamma$ (39). Cavnar et al. recently suggested that M1/M2 macrophage polarization is linked to oncogene activity in a mouse model of spontaneous GIST as well as in human GIST (40).

Several studies on patient material have brought attention to lymphocytes. Selected examples include a study describing high prevalence of tumor-infiltrating lymphocytes in STS (41). Others have shown that tumor-infiltrating CD3+ lymphocytes in GIST correlated with improved progression-free survival (42). Infiltration of CD8+ lymphocytes in Ewing sarcoma correlated with improved survival (43). Expression of immune checkpoint molecules, such as the $\mathrm{T}$ cell-suppressive receptor $\mathrm{PD}-1$, has been correlated with disease progression in, e.g., osteosarcoma (44). CD20+ lymphocytes have been associated with improved survival in a study on STS (45).

Sarcomas are often considered to be one-compartment tumors harboring limited activity from infiltrating fibroblast-like cells (25). PDGFR + stromal cells have been described in human rhabdomyosarcoma, where their presence showed a clinical association with subtype and metastasis (23). Frequently detected stromal components (osteoid, cartilage, or collagen) are however not likely attributed to infiltrating fibroblasts in the majority of sarcomas, but rather the sarcoma cells and/or the tissue-specific cells, themselves (46). Some investigators have suggested that stromal fibrosis/hyalinization is a specific pattern associated with a non-viable tumor following treatment in STS (47). 


\section{THERAPEUTIC TARGETING OF THE SARCOMA MICROENVIRONMENT}

There is currently a great enthusiasm for targeted therapies as a novel complementary or alternative treatment method in cancer. A number of potential molecular targets have also been discussed in the context of translational sarcoma studies (48). So far, most phase II and phase III clinical trials where a therapeutic benefit has been confirmed include studies on tyrosine kinase inhibitors (TKIs) targeting VEGFRs, PDGFRs, and KIT. Given the mesenchymal origin of sarcomas, these agents are likely to target both tumor cells and infiltrating stromal cells in a context-dependent manner (Figure 1). For more details, the reader is referred elsewhere $(36,49)$. Selected agents with presumed microenvironmental effects are described below.

\section{Therapies with Antiangiogenic Effects}

Angiogenesis was early predicted to be a common denominator for targeted therapy in a broad range of tumor types (17) and multiple studies have confirmed that antiangiogenic therapy causes starvation and reduced growth of experimental tumors. Accumulating evidence, however, suggest that the initial therapeutic benefit is followed by resistance mechanisms where the hypoxic and metabolic response to antiangiogenic therapy could worsen tumor aggressiveness (50).

One of the current antiangiogenic strategies is to alleviate hypoxia while improving tumor perfusion (51). Ideally, this approach increases response rates to radiotherapy, chemotherapy, and immunotherapy (5). Toxicity may however be a concern of some combination treatments. Of note is that the commonly targeted VEGFR pathway has not only been associated with induction of angiogenesis in STS but also with chemoresistance and regulatory $\mathrm{T}$ cell activity $(33,38)$.

The therapeutic efficacy of antiangiogenic therapy has been investigated in several sarcoma clinical trials. The TKI pazopanib was approved by the Food and Drug Administration (FDA) in 2012 for the use in advanced STS based on the results presented from the PALETTE phase III study (52). Anti-tumor activity has been demonstrated both in terms of sarcoma cell viability and inhibition of angiogenesis in a sarcoma cell line-dependent manner $(53,54)$.

Imatinib is another FDA-approved TKI with established ability to target KIT-mutated advanced GISTs. VEGF activity has, however, been described in GIST and imatinib can suppress GIST VEGF expression in vitro (55). Imatinib-responding GIST patients also display decreased serum VEGF levels. These antiangiogenic effects of imatinib, and other TKIs, have been reviewed by others (36). Only occasional responses to imatinib monotherapy have been observed in non-GIST sarcoma patients (56).

Sunitinib and regorafenib are both FDA-approved therapies for advanced GIST (second-, and third-line treatment, respectively) after failure to respond to imatinib. Their effects on tumor stroma, including angiogenesis, have not been clearly separated from the anti-proliferative effects on tumor cells (57-59).

\section{Therapies with Immune-Modulating Effects}

Several cancer therapies in sarcoma may have direct or indirect effects on the immune system $(38,60,61)$. Chemotherapy can, e.g., induce immunogenic cell death in tumors, block the

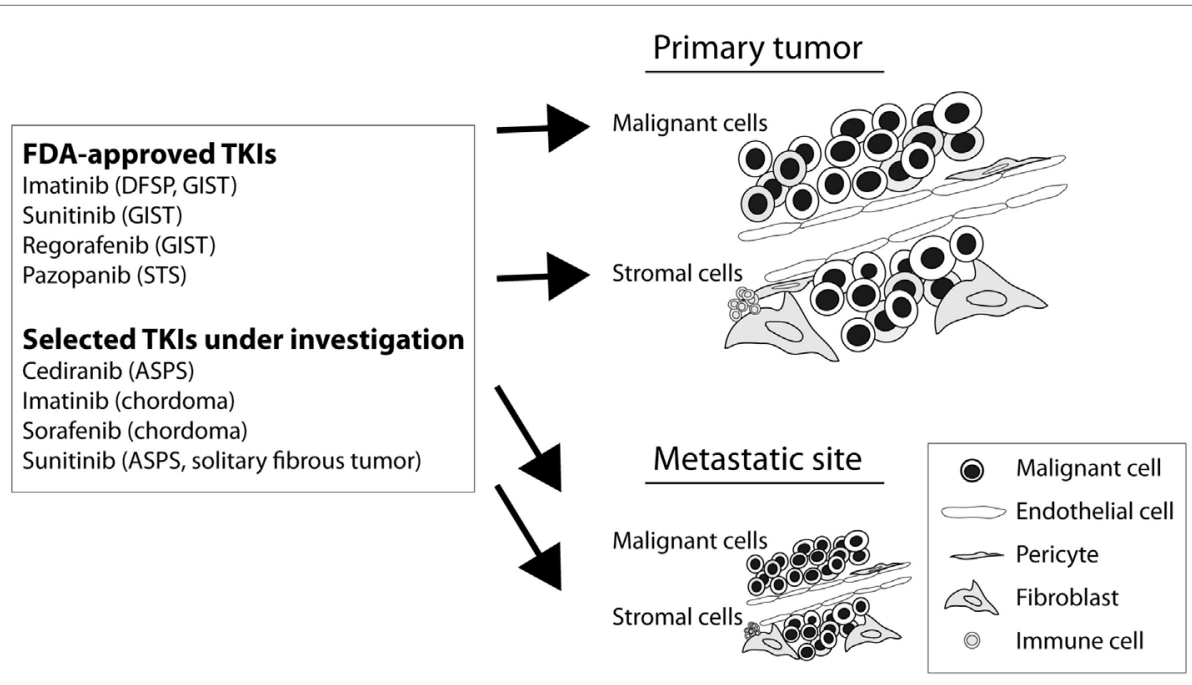

FIGURE 1 | Potential therapeutic effects of selected TKIs in sarcoma. The biological mechanisms associated with patient response to TKIs in sarcoma are generally poorly characterized. Imatinib has mainly been described for its ability to target aberrant PDGFR signaling in dermatofibrosarcoma protuberans (DFSP) and KIT (or PDGFR) in advanced GIST. Sunitinib is approved for second-line treatment of GIST, after imatinib treatment failure, with molecular targets including VEGFR, PDGFR, FLT3, KIT, CSF1R, and RET. Regorafenib is approved for third-line treatment of GIST, after sunitinib treatment failure, with molecular targets including VEGFR, KIT, RET, FGFR, PDGFR, and RAF. Pazopanib targets VEGFR, PDGFR, KIT, and RAF and is approved for use in advanced STS. Cediranib and sunitinib are investigated for their ability to target VEGFR signaling in, e.g., alveolar soft part sarcoma (ASPS). Imatinib and sorafenib are investigated for therapeutic use in chordoma, a disease with reported PDGFR activity. Sorafenib has a target spectrum, including RAF, VEGFR, PDGFR, KIT, RET, and FLT3. 
immunosuppressive functions of myeloid-derived suppressive cells and likely lead to a more pronounced anti-tumor response. Trabectedin, approved by the European Medicines Agency (EMA) for second-line treatment of advanced STS, is an example of a chemotherapeutic agent, with the additional ability to induce apoptosis selectively in monocytes/macrophages (62).

The EMA approved the immunostimulant mifamurtide in 2009 for the use in high-grade non-metastatic osteosarcoma in combination with postoperative chemotherapy. The treatment was shown to significantly improve 6-year overall survival from 70 to $78 \%$ (63). Additional studies on the therapeutic efficacy are however warranted $(2,63,64)$. Mifamurtide is reported to have its mechanism of action on macrophages and monocytes.

An illustrative example of a targeted therapy with immunemodulating side effects is imatinib treatment in advanced GIST, where an immunologic interferon- $\gamma$ response has been associated with long-term survival (65). The antiangiogenic, anti-tumor, and immunostimulating roles of interferons have been reviewed elsewhere (66).

Denosumab is an FDA-approved monoclonal antibody directed against RANKL, which is expressed by the neoplastic cells in giant-cell tumor of bone (67). Osteoclasts, their precursors and reactive osteoclast-like giant cells express the receptor RANK. RANKL-RANK signaling contributes to osteoclast formation, osteolysis, and tumor growth. This type of tumor is often benign, but with unpredictable behavior.

\section{Targeted Therapies Under Investigation}

Ongoing and future studies will reveal to what extent current targeted therapies under investigation have anti-tumor effects associated with the tumor microenvironment. Selected examples include the antiangiogenic TKIs sunitinib and cediranib with potential anti-tumor activity in ASPS, a malignancy associated with oncogenic MET signaling, pro-angiogenic factors, and inflammatory components $(38,68-70)$. Abundant VEGF expression has been confirmed. Sunitinib has also shown activity in solitary fibrous tumors (71). In the latter study, all cases were positive for PDGFR $\beta$ and/or VEGFR2.

Sorafenib is another widely used TKI with potential activity in subsets of sarcomas, either as mono- or combination therapy (72). Anti-tumor effects are believed to occur by several molecular mechanisms including inhibition of RAF, VEGFRs, PDGFRs, and KIT. Recently, the early results from a sorafenib phase II trial with locally advanced and metastatic chordoma patients were presented and compared with the results from two previous chordoma phase II trials with imatinib and lapatinib, respectively (73). Response rates were modest. Chordomas frequently express

\section{REFERENCES}

1. Mastrangelo G, Fadda E, Cegolon L, Montesco MC, Ray-Coquard I, Buja A, et al. A European project on incidence, treatment, and outcome of sarcoma. BMC Public Health (2010) 10:188. doi:10.1186/1471-2458-10-188

2. ESMO/European Sarcoma Network Working Group. Bone sarcomas: ESMO Clinical Practice Guidelines for diagnosis, treatment and growth factor receptors, such as PDGFRs and EGFRs (74), and VEGF expression has been confirmed $(75,76)$.

An example of an antibody-based targeted therapy with promising activity is the use of olaratumab, an anti-PDGFR $\alpha$ monoclonal antibody, in combination with doxorubicin in advanced STS (abstract 10501, ASCO Annual meeting 2015). In a randomized phase Ib/II study, an improvement of 10.3 months in overall survival was achieved compared to doxorubicin alone $(\mathrm{HR}=0.44, p=0.0005)$.

It is yet too early to say whether novel immune-modulating therapies will be of therapeutic value in sarcoma. Immune checkpoint inhibitors have emerged as a promising therapy in other tumor types and are currently being tested in sarcoma. T-cell receptor-based gene therapy directed against tumor-specific antigens is another type of treatment with promising activity in synovial sarcoma (77).

\section{CONCLUDING REMARKS}

Treatment options are today limited for many sarcoma patients. The infiltrative growth pattern of many sarcomas makes complete tumor resection with negative margins difficult. Distant metastases are often present already at diagnosis. Further preclinical and clinical studies are clearly needed to identify novel therapeutic targets.

The future directions of sarcoma diagnosis, therapy and followup are likely to rely more on tumor-specific biology. Careful monitoring of individual tumor genetics and gene/protein expression patterns is predicted to be essential. Therapy-adapted screening methods and standard criteria for tumor response assessment beyond the response evaluation criteria in solid tumors (RECIST) need to be further developed. Useful biomarkers, stromal components, and microenvironmental targets of importance for disease progression largely remain to be identified within the new era of personalized medicine.

\section{AUTHOR CONTRIBUTIONS}

ME wrote the manuscript, edited, and approved the final version to be published. OL critically revised the content of the work and approved the final version to be published.

\section{ACKNOWLEDGMENTS}

ME is financially supported by the Swedish Childhood Cancer Foundation, the Alex and Eva Wallström Foundation, and the Mary Béve Childhood Cancer Foundation. Arne Östman is acknowledged for his valuable comments on the manuscript.

follow-up. Ann Oncol (2014) 25(Suppl 3):iii113-23. doi:10.1093/ annonc/mdu256

3. ESMO/European Sarcoma Network Working Group. Soft tissue and visceral sarcomas: ESMO Clinical Practice Guidelines for diagnosis, treatment and follow-up. Ann Oncol (2014) 25(Suppl 3):iii102-12. doi:10.1093/annonc/ mdu254

4. ESMO/European Sarcoma Network Working Group. Gastrointestinal stromal tumours: ESMO Clinical Practice Guidelines for diagnosis, treatment 
and follow-up. Ann Oncol (2014) 25(Suppl 3):iii21-6. doi:10.1093/annonc/ mdu255

5. Wong P, Houghton P, Kirsch DG, Finkelstein SE, Monjazeb AM, Xu-Welliver $\mathrm{M}$, et al. Combining targeted agents with modern radiotherapy in soft tissue sarcomas. J Natl Cancer Inst (2014) 106(11):dju329. doi:10.1093/ jnci/dju329

6. Joensuu H, Hohenberger P, Corless CL. Gastrointestinal stromal tumour. Lancet (2013) 382(9896):973-83. doi:10.1016/S0140-6736(13)60106-3

7. Jo VY, Fletcher CD. WHO classification of soft tissue tumours: an update based on the 2013 (4th) edition. Pathology (2014) 46(2):95-104. doi:10.1097/ PAT. 0000000000000050

8. Fletcher CDM, Bridge JA, Hogendoorn PCW, Mertens F. WHO Classification of Tumours of Soft Tissue and Bone. Pathology and Genetics of Tumours of Soft Tissue and Bone. 4th ed. Lyon: IARC Press (2013).

9. Doyle LA. Sarcoma classification: an update based on the 2013 World Health Organization classification of tumors of soft tissue and Bone. Cancer (2014) 120(12):1763-74. doi:10.1002/cncr.28657

10. Helman LJ, Meltzer P. Mechanisms of sarcoma development. Nat Rev Cancer (2003) 3(9):685-94. doi:10.1038/nrc1168

11. Joensuu H, Rutkowski P, Nishida T, Steigen SE, Brabec P, Plank L, et al. KIT and PDGFRA mutations and the risk of GI stromal tumor recurrence. J Clin Oncol (2015) 33(6):634-42. doi:10.1200/JCO.2014.57.4970

12. Zwerner JP, May WA. PDGF-C is an EWS/FLI induced transforming growth factor in Ewing family tumors. Oncogene (2001) 20(5):626-33. doi:10.1038/ sj.onc. 1204133

13. Missiaglia E, Williamson D, Chisholm J, Wirapati P, Pierron G, Petel F, et al. PAX3/FOXO1 fusion gene status is the key prognostic molecular marker in rhabdomyosarcoma and significantly improves current risk stratification. $J$ Clin Oncol (2012) 30(14):1670-7. doi:10.1200/JCO.2011.38.5591

14. De Giovanni C, Landuzzi L, Nicoletti G, Lollini PL, Nanni P. Molecular and cellular biology of rhabdomyosarcoma. Future Oncol (2009) 5(9):1449-75. doi:10.2217/fon.09.97

15. Klein G, Sjogren HO, Klein E, Hellstrom KE. Demonstration of resistance against methylcholanthrene-induced sarcomas in the primary autochthonous host. Cancer Res (1960) 20:1561-72.

16. Joyce JA, Fearon DT. T cell exclusion, immune privilege, and the tumor microenvironment. Science (2015) 348(6230):74-80. doi:10.1126/science.aaa6204

17. Folkman J. Tumor angiogenesis: therapeutic implications. N Engl J Med (1971) 285(21):1182-6. doi:10.1056/NEJM197111182852108

18. Paget S. The distribution of secondary growths in cancer of the breast. 1889. Cancer Metastasis Rev (1989) 8(2):98-101.

19. Ewing J. Neoplastic Diseases: A Treatise on Tumors. 3rd ed., rev., and enl., with 546 Illustrations. Philadelphia, PA: W. B. Saunders (1928). 1127 p.

20. Langley RR, Fidler IJ. The seed and soil hypothesis revisited - the role of tumor-stroma interactions in metastasis to different organs. Int $J$ Cancer (2011) 128(11):2527-35. doi:10.1002/ijc.26031

21. Sugarbaker EV. Cancer metastasis: a product of tumor-host interactions. Curr Probl Cancer (1979) 3(7):1-59. doi:10.1016/S0147-0272(79)80008-2

22. Engellau J, Bendahl PO, Persson A, Domanski HA, Akerman M, Gustafson P, et al. Improved prognostication in soft tissue sarcoma: independent information from vascular invasion, necrosis, growth pattern, and immunostaining using whole-tumor sections and tissue microarrays. Hum Pathol (2005) 36(9):994-1002. doi:10.1016/j.humpath.2005.07.008

23. Ehnman M, Missiaglia E, Folestad E, Selfe J, Strell C, Thway K, et al. Distinct effects of ligand-induced PDGFRalpha and PDGFRbeta signaling in the human rhabdomyosarcoma tumor cell and stroma cell compartments. Cancer Res (2013) 73(7):2139-49. doi:10.1158/0008-5472.CAN-12-1646

24. Pardali E, van der Schaft DW, Wiercinska E, Gorter A, Hogendoorn PC, Griffioen AW, et al. Critical role of endoglin in tumor cell plasticity of Ewing sarcoma and melanoma. Oncogene (2011) 30(3):334-45. doi:10.1038/ onc. 2010.418

25. Tomlinson J, Barsky SH, Nelson S, Singer S, Pezeshki B, Lee MC, et al. Different patterns of angiogenesis in sarcomas and carcinomas. Clin Cancer Res (1999) 5(11):3516-22.

26. West CC, Brown NJ, Mangham DC, Grimer RJ, Reed MW. Microvessel density does not predict outcome in high grade soft tissue sarcoma. Eur J Surg Oncol (2005) 31(10):1198-205. doi:10.1016/j.ejso.2005.04.012
27. Imamura M, Yamamoto H, Nakamura N, Oda Y, Yao T, Kakeji Y, et al. Prognostic significance of angiogenesis in gastrointestinal stromal tumor. Mod Pathol (2007) 20(5):529-37. doi:10.1038/modpathol.3800767

28. Ahlen J, Wejde J, Brosjo O, von Rosen A, Weng WH, Girnita L, et al. Insulinlike growth factor type 1 receptor expression correlates to good prognosis in highly malignant soft tissue sarcoma. Clin Cancer Res (2005) 11(1):206-16.

29. Kawauchi S, Fukuda T, Tsuneyoshi M. Angiogenesis does not correlate with prognosis or expression of vascular endothelial growth factor in synovial sarcomas. Oncol Rep (1999) 6(5):959-64.

30. Lei P, Ding D, Xie J, Wang L, Liao Q, Hu Y. Expression profile of twist, vascular endothelial growth factor and CD34 in patients with different phases of osteosarcoma. Oncol Lett (2015) 10(1):417-21. doi:10.3892/ol.2015.3246

31. Ohsawa M, Tomita Y, Kuratsu S, Kanno H, Aozasa K. Angiogenesis in malignant fibrous histiocytoma. Oncology (1995) 52(1):51-4. doi:10.1159/000227427

32. Saenz NC, Heslin MJ, Adsay V, Lewis JJ, Leung DH, LaQuaglia MP, et al. Neovascularity and clinical outcome in high-grade extremity soft tissue sarcomas. Ann Surg Oncol (1998) 5(1):48-53. doi:10.1007/BF02303764

33. Sleijfer S, van der Graaf WT, Blay JY. Angiogenesis inhibition in non-GIST soft tissue sarcomas. Oncologist (2008) 13(11):1193-200. doi:10.1634/ theoncologist.2008-0188

34. Takahashi R, Tanaka S, Kitadai Y, Sumii M, Yoshihara M, Haruma K, et al. Expression of vascular endothelial growth factor and angiogenesis in gastrointestinal stromal tumor of the stomach. Oncology (2003) 64(3):266-74. doi: $10.1159 / 000069316$

35. DuBois S, Demetri G. Markers of angiogenesis and clinical features in patients with sarcoma. Cancer (2007) 109(5):813-9. doi:10.1002/cncr.22455

36. Versleijen-Jonkers YM, Vlenterie $M$, van de Luijtgaarden AC, van der Graaf WT. Anti-angiogenic therapy, a new player in the field of sarcoma treatment. Crit Rev Oncol Hematol (2014) 91(2):172-85. doi:10.1016/j. critrevonc.2014.02.001

37. Lee TH, Bolontrade MF, Worth LL, Guan H, Ellis LM, Kleinerman ES. Production of VEGF165 by Ewing's sarcoma cells induces vasculogenesis and the incorporation of $\mathrm{CD} 34+$ stem cells into the expanding tumor vasculature. Int J Cancer (2006) 119(4):839-46. doi:10.1002/ijc.21916

38. Castelli C, Rivoltini L, Rodolfo M, Tazzari M, Belgiovine C, Allavena P. Modulation of the myeloid compartment of the immune system by angiogenic- and kinase inhibitor-targeted anti-cancer therapies. Cancer Immunol Immunother (2015) 64(1):83-9. doi:10.1007/s00262-014-1576-1

39. O'Sullivan T, Saddawi-Konefka R, Vermi W, Koebel CM, Arthur C, White JM, et al. Cancer immunoediting by the innate immune system in the absence of adaptive immunity. J Exp Med (2012) 209(10):1869-82. doi:10.1084/ jem. 20112738

40. Cavnar MJ, Zeng S, Kim TS, Sorenson EC, Ocuin LM, Balachandran VP, et al. KIT oncogene inhibition drives intratumoral macrophage M2 polarization. $J$ Exp Med (2013) 210(13):2873-86. doi:10.1084/jem.20130875

41. D’Angelo SP, Shoushtari AN, Agaram NP, Kuk D, Qin LX, Carvajal RD, et al. Prevalence of tumor-infiltrating lymphocytes and PD-L1 expression in the soft tissue sarcoma microenvironment. Hum Pathol (2015) 46(3):357-65. doi:10.1016/j.humpath.2014.11.001

42. Rusakiewicz S, Semeraro M, Sarabi M, Desbois M, Locher C, Mendez R, et al. Immune infiltrates are prognostic factors in localized gastrointestinal stromal tumors. Cancer Res (2013) 73(12):3499-510. doi:10.1158/0008-5472. CAN-13-0371

43. Berghuis D, Santos SJ, Baelde HJ, Taminiau AH, Egeler RM, Schilham MW, et al. Pro-inflammatory chemokine-chemokine receptor interactions within the Ewing sarcoma microenvironment determine CD8(+) T-lymphocyte infiltration and affect tumour progression. J Pathol (2011) 223(3):347-57. doi:10.1002/path.2819

44. Zheng W, Xiao H, Liu H, Zhou Y. Expression of programmed death 1 is correlated with progression of osteosarcoma. APMIS (2015) 123(2):102-7. doi:10.1111/apm.12311

45. Sorbye SW, Kilvaer T, Valkov A, Donnem T, Smeland E, Al-Shibli K, et al. Prognostic impact of lymphocytes in soft tissue sarcomas. PLoS One (2011) 6(1):e14611. doi:10.1371/journal.pone.0014611

46. Picci P, Rougraff BT, Bacci G, Neff JR, Sangiorgi L, Cazzola A, et al. Prognostic significance of histopathologic response to chemotherapy in nonmetastatic Ewing's sarcoma of the extremities. J Clin Oncol (1993) 11(9):1763-9. 
47. Lucas DR, Kshirsagar MP, Biermann JS, Hamre MR, Thomas DG, Schuetze SM, et al. Histologic alterations from neoadjuvant chemotherapy in high-grade extremity soft tissue sarcoma: clinicopathological correlation. Oncologist (2008) 13(4):451-8. doi:10.1634/theoncologist.2007-0220

48. Yang J, Zhang W. New molecular insights into osteosarcoma targeted therapy. Curr Opin Oncol (2013) 25(4):398-406. doi:10.1097/CCO.0b013e3283622c1b

49. Ehnman M, Ostman A. Therapeutic targeting of platelet-derived growth factor receptors in solid tumors. Expert Opin Investig Drugs (2014) 23(2):211-26. doi:10.1517/13543784.2014.847086

50. McIntyre A, Harris AL. Metabolic and hypoxic adaptation to anti-angiogenic therapy: a target for induced essentiality. EMBO Mol Med (2015) 7(4):368-79. doi:10.15252/emmm.201404271

51. Jain RK. Antiangiogenesis strategies revisited: from starving tumors to alleviating hypoxia. Cancer Cell (2014) 26(5):605-22. doi:10.1016/j.ccell.2014.10.006

52. van der Graaf WT, Blay JY, Chawla SP, Kim DW, Bui-Nguyen B, Casali PG, et al. Pazopanib for metastatic soft-tissue sarcoma (PALETTE): a randomised, double-blind, placebo-controlled phase 3 trial. Lancet (2012) 379(9829):1879-86. doi:10.1016/S0140-6736(12)60651-5

53. Kumar S, Mokhtari RB, Sheikh R, Wu B, Zhang L, Xu P, et al. Metronomic oral topotecan with pazopanib is an active antiangiogenic regimen in mouse models of aggressive pediatric solid tumor. Clin Cancer Res (2011) 17(17):5656-67. doi:10.1158/1078-0432.CCR-11-0078

54. Li H, Wozniak A, Sciot R, Cornillie J, Wellens J, Van Looy T, et al. Pazopanib, a receptor tyrosine kinase inhibitor, suppresses tumor growth through angiogenesis in dedifferentiated liposarcoma xenograft models. Transl Oncol (2014) 7(6):665-71. doi:10.1016/j.tranon.2014.09.007

55. Jin T, Nakatani H, Taguchi T, Nakano T, Okabayashi T, Sugimoto T, et al. STI571 (Glivec) suppresses the expression of vascular endothelial growth factor in the gastrointestinal stromal tumor cell line, GIST-T1. World J Gastroenterol (2006) 12(5):703-8. doi:10.3748/wjg.v12.i5.703

56. Chugh R, Wathen JK, Maki RG, Benjamin RS, Patel SR, Meyers PA, et al. Phase II multicenter trial of imatinib in 10 histologic subtypes of sarcoma using a Bayesian hierarchical statistical model. J Clin Oncol (2009) 27(19):3148-53. doi:10.1200/JCO.2008.20.5054

57. Demetri GD, van Oosterom AT, Garrett CR, Blackstein ME, Shah MH, Verweij J, et al. Efficacy and safety of sunitinib in patients with advanced gastrointestinal stromal tumour after failure of imatinib: a randomised controlled trial. Lancet (2006) 368(9544):1329-38. doi:10.1016/ S0140-6736(06)69446-4

58. George S, Wang Q, Heinrich MC, Corless CL, Zhu M, ButrynskiJE, et al. Efficacy and safety of regorafenib in patients with metastatic and/or unresectable GI stromal tumor after failure of imatinib and sunitinib: a multicenter phase II trial. J Clin Oncol (2012) 30(19):2401-7. doi:10.1200/JCO.2011.39.9394

59. Demetri GD, Reichardt P, Kang YK, Blay JY, Rutkowski P, Gelderblom H, et al. Efficacy and safety of regorafenib for advanced gastrointestinal stromal tumours after failure of imatinib and sunitinib (GRID): an international, multicentre, randomised, placebo-controlled, phase 3 trial. Lancet (2013) 381(9863):295-302. doi:10.1016/S0140-6736(12)61857-1

60. Burgess M, Tawbi H. Immunotherapeutic approaches to sarcoma. Curr Treat Options Oncol (2015) 16(6):26. doi:10.1007/s11864-015-0345-5

61. Zitvogel L, Galluzzi L, Smyth MJ, Kroemer G. Mechanism of action of conventional and targeted anticancer therapies: reinstating immunosurveillance. Immunity (2013) 39(1):74-88. doi:10.1016/j.immuni.2013.06.014

62. Germano G, Frapolli R, Belgiovine C, Anselmo A, Pesce S, Liguori M, et al. Role of macrophage targeting in the antitumor activity of trabectedin. Cancer Cell (2013) 23(2):249-62. doi:10.1016/j.ccr.2013.01.008

63. Meyers PA, Schwartz CL, Krailo MD, Healey JH, Bernstein ML, Betcher D, et al. Osteosarcoma: the addition of muramyl tripeptide to chemotherapy improves overall survival - a report from the Children's Oncology Group. J Clin Oncol (2008) 26(4):633-8. doi:10.1200/JCO.2008.14.0095
64. Hunsberger S, Freidlin B, Smith MA. Complexities in interpretation of osteosarcoma clinical trial results. J Clin Oncol (2008) 26(18):3103-4. doi:10.1200/ JCO.2008.17.3484 author reply 4-5,

65. Menard C, Blay JY, Borg C, Michiels S, Ghiringhelli F, Robert C, et al. Natural killer cell IFN-gamma levels predict long-term survival with imatinib mesylate therapy in gastrointestinal stromal tumor-bearing patients. Cancer Res (2009) 69(8):3563-9. doi:10.1158/0008-5472.CAN-08-3807

66. Whelan J, Patterson D, Perisoglou M, Bielack S, Marina N, Smeland S, et al. The role of interferons in the treatment of osteosarcoma. Pediatr Blood Cancer (2010) 54(3):350-4. doi:10.1002/pbc.22136

67. Chawla S, Henshaw R, Seeger L, Choy E, Blay JY, Ferrari S, et al. Safety and efficacy of denosumab for adults and skeletally mature adolescents with giant cell tumour of bone: interim analysis of an open-label, parallel-group, phase 2 study. Lancet Oncol (2013) 14(9):901-8. doi:10.1016/ S1470-2045(13)70277-8

68. Kummar S, Allen D, Monks A, Polley EC, Hose CD, Ivy SP, et al. Cediranib for metastatic alveolar soft part sarcoma. J Clin Oncol (2013) 31(18):2296-302. doi:10.1200/JCO.2012.47.4288

69. Stacchiotti S, Tamborini E, Marrari A, Brich S, Rota SA, Orsenigo M, et al. Response to sunitinib malate in advanced alveolar soft part sarcoma. Clin Cancer Res (2009) 15(3):1096-104. doi:10.1158/1078-0432.CCR-08-2050

70. Lazar AJ, Lahat G, Myers SE, Smith KD, Zou C, Wang WL, et al. Validation of potential therapeutic targets in alveolar soft part sarcoma: an immunohistochemical study utilizing tissue microarray. Histopathology (2009) 55(6):750-5 doi:10.1111/j.1365-2559.2009.03436.x

71. Stacchiotti S, Negri T, Libertini M, Palassini E, Marrari A, De Troia B, et al. Sunitinib malate in solitary fibrous tumor (SFT). Ann Oncol (2012) 23(12):3171-9. doi:10.1093/annonc/mds143

72. Gadaleta-Caldarola G, Infusino S, Divella R, Ferraro E, Mazzocca A, De Rose F, et al. Sorafenib: 10 years after the first pivotal trial. Future Oncol (2015) 11(13):1863-80. doi:10.2217/fon.15.85

73. Bompas E, Le Cesne A, Tresch-Bruneel E, Lebellec L, Laurence V, Collard O, et al. Sorafenib in patients with locally advanced and metastatic chordomas: a phase II trial of the French Sarcoma Group (GSF/GETO). Ann Oncol (2015) 26(10):2168-73. doi:10.1093/annonc/mdv300

74. Fasig JH, Dupont WD, LaFleur BJ, Olson SJ, Cates JM. Immunohistochemical analysis of receptor tyrosine kinase signal transduction activity in chordoma. Neuropathol Appl Neurobiol (2008) 34(1):95-104. doi:10.1111/j.1365-2990.2007.00873.x

75. Li X, Ji Z, Ma Y, Qiu X, Fan Q, Ma B. Expression of hypoxia-inducible factor-1alpha, vascular endothelial growth factor and matrix metalloproteinase-2 in sacral chordomas. Oncol Lett (2012) 3(6):1268-74. doi:10.3892/ol.2012.645

76. Chen KW, Yang HL, Lu J, Wang GL, Ji YM, Wu GZ, et al. Expression of vascular endothelial growth factor and matrix metalloproteinase-9 in sacral chordoma. J Neurooncol (2011) 101(3):357-63. doi:10.1007/s11060-010-0263-0

77. Robbins PF, Morgan RA, Feldman SA, Yang JC, Sherry RM, Dudley ME, et al. Tumor regression in patients with metastatic synovial cell sarcoma and melanoma using genetically engineered lymphocytes reactive with NY-ESO-1. J Clin Oncol (2011) 29(7):917-24. doi:10.1200/JCO.2010.32.2537

Conflict of Interest Statement: The authors declare that the research was conducted in the absence of any commercial or financial relationships that could be construed as a potential conflict of interest.

Copyright (c) 2015 Ehnman and Larsson. This is an open-access article distributed under the terms of the Creative Commons Attribution License (CC BY). The use, distribution or reproduction in other forums is permitted, provided the original author(s) or licensor are credited and that the original publication in this journal is cited, in accordance with accepted academic practice. No use, distribution or reproduction is permitted which does not comply with these terms. 